\title{
Secuencia y delimitación en el procesamiento textual alemán: modelo conexionista.
}

\author{
Carmen-Cayetana Castro Moreno \\ Universidad de Sevilla \\ ccastro1@us.es \\ https://dx.doi.org/10.12795/futhark.2012.i07.03
}

\begin{abstract}
In order to simulate human speech, connectionism has developed several techniques for representation of frames and scripts. These models are able to solve some of the shortcomings of classical cognitive systems. Specifically, classical systems are unable to control when a chunk of knowledge has to be activated and what part of the chunk should be more activated. Instead, connectionist systems can use degrees in mobilizing to try this case. However, connectionism has also some limitations if considered as models of human mind, but they also are a point of interest to the application in the prosecution textual. Besides, we examinate the plausibility of any of these approaches used in computers and their implications in our own connectionist model.
\end{abstract}

Keywords: Connectionism, Schema, Knowledge representation, Discourse processing

Resumen: El conexionismo, emulando el procesamiento de discursivo, ha llevado a cabo el desarrollado de variadas técnicas en la representación de frames y scripts. Estos modelos son capaces de solucionar algunas de las limitaciones de los sistemas clásicos cognitivos. Especialmente, los sistemas clásicos no disponen de la capacidad de activar un chunk de conocimiento y qué parte de ese chunk ha de estar más activada. En cambio, los sistemas conexionistas pueden utilizar grados de activación para tratar este caso. No obstante, estos modelos tienen alguna limitación al intentar reproducir la mente humana, si bien contemplan aspectos de interés para la aplicación en el procesamiento textual. Además, es analizada la plausibidad de algunos de estos enfoques empleados en computación y se implican en nuestro modelo conexionista.

Palabras clave: Conexionismo, Esquema, Representación del conocimiento, Procesamiento de discurso

\section{Introducción a la teoría cognitiva}

La teoría cognitiva tiene una historia muy reciente, si bien sus primeras propuestas se remontan a finales de la década de los 40 y principios de los 50, y tan sólo a partir de los años 60 se empieza a extender por el mundo entero'. No obstante, en esos

\footnotetext{
${ }^{1}$ El inmediato antecesor de la psicología cognitiva, que nos ocupa para explicar fenómenos lingüísticos, y al que nos vamos a remontar escuetamente, es el conductismo, que destacó por los siguientes rasgos fundamentales: - El conductismo
} 
años los dos argumentos fundamentales del conductismo, desde un punto de vista psicológico, el ambientalismo y el organismo vacío comienzan a entrar en decadencia ${ }^{2}$.

Surge así la psicología cognitiva, la cual difiere de otros métodos como el mentalismo, con otras palabras conocido como conductismo metodológico, según el cual, y tras las reformulaciones de Shastri y Ajjanagade (cf. 2006: 417-494), la investigación psicológica debe basarse en la observación y experimentación de la conducta. La principal novedad es que comenzó a estudiarse la "mente" como sistema capaz de manipular información, y aspectos como la motivación y la emoción quedaban dentro de su campo de estudio ${ }^{3}$.

Las ideas filosóficas racionalistas juegan un papel primordial en la manera de interpretarse la cognición, pues consideran que la mente actúa siguiendo unas reglas, como pueden ser las tecnológicas, parecidas a las atribuidas al ordenador de Von Neumann, el cual operaba mediante un procedimiento serial, parecido al de los ordenadores actuales, y cada una de sus diferentes partes tenían atribuidas funciones concretas. Así en 1986 D. E. Rumelhart y J. L. McClelland publican la obra "Procesamiento Distribuido en Paralelo", de la que surge una novedosa forma de entender la cognición, el conexionismo, que por la crítica es considerado como un nuevo paradigma (cf. Rumelhart 1996) ${ }^{4}$.

es una ciencia sin sujeto, es decir, lo único importante es la estimulación ambiental, las conductas que son reforzadas, el ambiente en el que vive el sujeto, por tanto todos los sujetos en esencia pueden ser considerados iguales, las diferencias genéticas o carecen de importancia o son ignoradas. - El conductismo también rechaza la mente (o cognición), por lo que todo se reduce a un conjunto de estímulos y respuestas que operan mecánicamente. El conductismo estudia la conducta para explicarla.

2 La etología, la neurociencia, los desarrollos de la propia psicología traen consigo una vuelta a los antiguos temas de la psicología mentalista pero con una perspectiva metodológica nueva. Nace así la psicología cognitiva con el objeto de estudiar la mente mirando la conducta.

3 Cuando nació la psicología cognitiva esta solo se ocupaba de los estados informacionales, o sea, de la dimensión intelectual, por lo que supone un estado informacional nuevo. La teoría conexionista de computación incluye entre otras, la lógica borrosa, las redes neuronales y el razonamiento aproximado.

${ }^{4}$ Entre la psicología cognitiva clásica y el conexionismo o procesamiento distribuido en paralelo hay similitudes y diferencias que se puede resumir en: 1) Computación:

Futhark 7 (2012)

Castro, Secuencia y delimitación, 85-114

ISSN $1886-9300$ 
En su esencia psicolingüística, el fenómeno de la adquisición del lenguaje ya ha sido abordado a través de las teorías más variadas, desde sus enfoques más conductistas hasta los conexionistas, los cuales han intentado aportar soluciones a los problemas que se describían, así como a los procesos que involucraban. Pues bien, estas teorías que rozan en la mayoría de los casos el terreno de la Psicología como ciencia, no han logrado hasta el momento dar cuenta de la totalidad de dichos procesos.

Por nuestra parte, pensamos que acotar los enfoques que en su campo más representativo tratan de explicar cómo se organiza el conocimiento alrededor de determinados conceptos, acontecimientos o eventos almacenados en la memoria a largo plazo, (MLP), podría aportar a su vez nuevos datos a nuestro modelo explicativo de procesamiento textual, basado en sus orígenes en la adquisición del lenguaje, y nos facilitaría la comprensión de diversos modelos textuales. A partir de estos modelos textuales se analizan determinadas secuencias y se delimita la perspectiva conexionista aplicándose a procedimientos traslativos y discursivos en lengua alemana y española.

\section{Conexión, adquisición, producción}

En ocasiones se prefiere llamar al "conexionismo" también "neoconexionismo", para diferenciarlo de aquella forma de conexionismo que ya propuso Alexander Bain en la segunda mitad del siglo XIX ${ }^{5}$. El conexionismo también es conocido como

para ambos modelos teóricos la computación en el núcleo central de ambas teorías, sin embargo cada uno concibe la computación de maneras muy distintas: 2) Objetivos: para la psicología cognitiva clásica el objetivo es descubrir las reglas, elementos y estructuras básicas que rigen el procesamiento humano. Para el conexionismo es el descubrimiento de redes adecuadas de simulación y reglas de aprendizaje eficientes. 3) Aprendizaje: La psicología cognitiva clásica se mantiene en posturas innatistas y rechaza explícitamente el asociacionismo, mientras que el conexionismo en cierto sentido es una forma de asociacionismo y mantiene la idea de mente como una "tabula rasa" en la que el ambiente va modelando los pesos de conexión de la red. Para algunos estas diferencias separan al conexionismo de la psicología cognitiva y lo aproximan al conductismo, dado que este defiende asociacionismo y ambientalismo.

${ }^{5}$ Este autor destacó desde un primer momento la importancia de las conexiones entre neuronas y la investigación y experimentación fisiológica.

Futhark 7 (2012)

Castro, Secuencia y delimitación, 85-114

ISSN $1886-9300$ 
procesamiento distribuido en paralelo (PDP). La teoría conexionista establece que la adquisición del lenguaje es más efectiva a través de la exposición a su lengua materna: el ser humano capta la información y la procesa a través de conexiones neuronales cerebrales. Estas teorías de conexión intentan simular el pensamiento humano en la computación, aproximándose a los sistemas biológicos.

Partiendo del los presupuestos conexionistas, los cuales emplean redes neuronales para comprender y explicar la vida psíquica y la conducta, entendemos las redes neuronales como un conjunto de unidades interconectadas masivamente capaces de procesar y almacenar información mediante la modificación de sus estados (cf. Crespo 2002). Aunque con matices, desde la psicolingüística, que analiza el proceso mental de producción del lenguaje, se considera que cuando los seres humanos realizan un determinado acto de habla, el fenómeno psicológico puede ser explicado según el modelo neuronal que construyen las teorías de conexión, creándose así un "modelo conexionista", aplicable a otras ramas de la Lingüística, tales como la contrastiva. La lingüística contrastiva es de especial interés en ejemplificaciones textuales pues aporta en su dimensión traductológica un acercamiento a las lenguas en cuestión, y evita los ya conocidos errores de doble codificación e interferencias del traductor.

Lange y Dyer (2009: 303-318) hacen mención a los elementos característicos presentes en la mayoría de los plantemientos conexionistas. Éstos incluyen el procedimiento textual en su conjunto, con sus componentes de producción y comprensión:

1. La red es un conjunto de unidades de procesamiento muy simples.

2. Dichas unidades interactúan mediante conexiones que los asocian.

3. Los estímulos que afectan a las unidades de entrada se expresan en términos cuantitativos.

4. Toda unidad de la red viene definida por un nivel de activación expresado de forma cuantitativa. 
5. Toda conexión se caracteriza por un valor de fuerza del trazo o peso de conexión, también expresado de forma cuantitativa.

6. El procesamiento y almacenamiento de la información se realiza de manera distribuida y paralela.

7. Existen ciertas reglas o funciones que modifican el nivel de activación a partir de las entradas para la producción de una salida a partir de un nivel de activación.

8. Existen ciertas reglas o funciones de aprendizaje que permiten a la red modificar los pesos de conexiones para acomodar de modo más perfecto la información de entrada a la salida requerida.

9. La función de cada unidad es simplemente realizar cálculos con las entradas que recibe y producir una salida a las unidades con las que está conectada.

10. Las señales de salida se expresan en términos cuantitativos.

11. Toda red neural es un dispositivo que permite computar una función, es decir, transformar una señal de entrada en una señal de salida. La función presente en la red y que realiza el cómputo es básicamente el patrón o conjunto de pesos sinápticos de las unidades."

Quizá se deba de advertir al lector de que las teorías conexionistas están generalmente vinculadas con los antecedentes fundantes de la Escuela Lingüística de Valparaíso, ELV, (cf. Parodi, Venegas, Ibáñez, y Gutiérrez 2008: 39-74).

Presenta y defiende esta Escuela una tesis teórico-empírica que puede resultar obvia para muchos investigadores fuera del ámbito estricto de la lingüística (tales como psicolingüistas, cientistas cognitivos, psicólogos evolutivos y psicólogos del discurso) en que se tiende a acoger una mirada inter y transdisciplinaria, que podría resultar controversial y no evidente para un número significativo de investigadores desde la teoría de la comunicación, la sociología, el análisis del discurso, el análisis crítico del discurso y, en general, desde ciertas áreas de las humanidades, de las ciencias sociales y de las ciencias computacionales. 


\subsection{Límites de memoria y círculos de comprensión}

Limitándonos a exponer nuestro modelo, para el que tomamos como ejemplo a McClelland y Rumelhart (1996), obtenemos que las representaciones y los procesos estarían distribuidos en un conjunto amplio de unidades neuronales simples: el ejemplo más significativo consistiría en que la actividad inteligente surge de una masa grande e interconectada de unidades neuronales simples. $\mathrm{Y}$ son estas unidades las que se conectan mediante vínculos externos ${ }^{6}$.

Si en nuestro primer texto, "El viaje" / "Die Reise" tuviéramos además en cuenta la presuposición de las teorías simbólicas (las cuales afirman que existe una memoria de trabajo y un conjunto amplio de conceptos, proposiciones, esquemas, y a su vez numerosas reglas de producción -con formato si-entonces-), llegaríamos a la conclusión de que "si los pasajeros no hubieran gritado a tiempo", en última consecuencia "el avión se habría estrellado". Se establece así un límite de memoria que identifica "la buena ocurrencia": "los pasajeros otras veces habían gritado con más tiempo". Aquí obtenemos la prueba de que la información en la memoria de trabajo varía a lo largo del tiempo, o expresado de otra forma, de ciclo de comprensión en ciclo de comprensión. De ello se deriva que el sistema se aprenda de estos cambios dinámicos. Consideraríamos entonces que cuando se dan las condiciones necesarias, la producción se activa y la acción o secuencia de acciones se realiza o no. El texto determina nuestras afirmaciones:

\section{[Texto 1]}

Texto en lengua de destino o L2 - "Die Reise"

\footnotetext{
${ }^{6}$ A la hora de enfrentamos con la difícil tarea de comprender los presupuestos conexionistas, desarrollados básicamente, en el campo de la psicología con el objeto de aclarar habilidades y competencias mentales (percepción, lenguaje, pensamiento...) nos enfrentamos a modelos de la mente. Por el contrario, hay ejemplos de redes empleadas en el ámbito de la neurociencia y que describen procesos únicamente cerebrales, caso en el que seguimos modelos del cerebro.

Futhark 7 (2012)

Castro, Secuencia y delimitación, 85-114
}

ISSN 1886-9300 
Die Fluggäste sitzen angeschnallt auf ihren Plätzen und warten auf die Piloten für den Start. In diesem Moment betreten zwei Männer in Pilotenuniform das Flugzeug: Einer wird von einem Blindenhund begleitet und der Andere sucht sich seinen Weg mit Hilfe eines weißen Stocks. Sie gehen ins Cockpit und schließen die Tür.

Einige Passagiere lachen nervös und alle schauen sich mit einem Ausdruck zwischen Erstaunen, Angst und Skepsis an. Einen Augenblick später springen die Motoren an und das Flugzeug beschleunigt auf der Piste. Es wird immer schneller und scheint offenbar nie abzuheben. Die Passagiere schauen aus den Luken und bemerken, dass das Flugzeug direkt auf einen See am Ende der Startbahn zusteuert. Das Flugzeug fährt nun sehr schnell über die Piste, immer näher auf den See zu und einige Reisende glauben schon, sie würden niemals abheben und das Flugzeug würde in dem See enden. Die Schreie der verängstigten Passagiere durchdringen das Flugzeug, aber genau in diesem Moment hebt die Maschine langsam und ohne Probleme ab. Die Pasagiere beruhigen sich sogleich, lachen und halten sich für dumm, weil sie sich so erschreckt haben. Nach ein paar Minuten ist der Vorfall vergessen. Das Kabinenpersonal bietet Wein an: Ich möchte nichts, danke- sagt ein junges Mädchen, - ich trinke nicht mehr.

Im Cockpit tastet der Pilot über die Instrumententafel, findet den Knopf für den Autopiloten und betätigt inn. Darauf sagt der Copilot: Eines Tages werden diese A. (Arschlöcher) zu spät schreien und wir gehen alle drauf...

\footnotetext{
${ }^{7}$ Texto en lengua original o L1 - "El Viaje": Los pasajeros de un avión están instalados en sus asientos y esperan a los pilotos para el despegue. En ese momento, dos hombres entran en el avión, con uniforme de piloto: llevan gafas negras. Uno de ellos va acompañado de un perro lazarillo y el otro va abriéndose camino ayudado de un bastón blanco. Entran en la cabina de pilotaje y cierran la puerta. Algunos pasajeros se ríen nerviosamente y todos se miran con una expresión entre sorpresa, miedo y escepticismo. Un momento después, se encienden los motores del avión y el avión empieza a tomar velocidad en la pista. Va cada vez más deprisa y parece no despegar nunca. Los pasajeros miran por las ventanillas y se dan cuenta que el avión se dirige directamente hacía el lago que se encuentra al final de la pista. El avión va ahora muy rápido sobre la pista, cada vez más cerca del lago, y varios viajeros empiezan a pensar que nunca despegarán y que el avión va a acabar en el lago. Los gritos de los pasajeros asustados llenan entonces el avión, pero justo en ese Futhark 7 (2012)

Castro, Secuencia y delimitación, 85-114
}

ISSN $1886-9300$ 
De lo anterior deducimos que el proceso de aprender puede crear nuevos hechos y reglas de producción en la memoria a largo plazo, los cuáles podrían ser relevantes a la hora de su traducción. En nuestro modelo conexionista, los condicionantes adquieren determinados parámetros: los vínculos denominados externos, se conforman mediante redes neuronales de tipo excitatorio o positivo, inhibitorio o negativo y neutro $o$ indiferente. Un ejemplo de distribución -aplicable a computación- podría ser el de la siguiente ejemplificación:

- Excitatorios o positivos: p. ej. "en ese momento, dos hombres entran en el avión, con uniforme de piloto: llevan gafas negras" / "Un momento después, se encienden los motores del avión y el avión empieza a tomar velocidad en la pista..."

- Inhibitorios o negativos: "Uno de ellos va acompañado de un perro lazarillo y el otro va abriéndose camino ayudado de un bastón blanco / Algunos pasajeros se ríen nerviosamente y todos se miran con una expresión entre sorpresa, miedo y escepticismo / Va cada vez más deprisa y parece no despegar nunca..."

- Neutros o indiferentes, o que no implican necesariamente efecto/reacción, p. ej.: "Los pasajeros de un avión están instalados en sus asientos y esperan a los pilotos para el despegue / "Entran en la cabina de pilotaje y cierran la puerta..."

Advertimos cómo estos parámetros en su vertiente positiva y neutra se realizan con un mayor índice de frecuencia en expresiones de negación y se asume, por tanto, que la comprensión se basa en elementos representacionales que reflejan los rasgos de las situaciones del mundo real descritas en el texto:

momento, el avión despega lentamente, sin ningún problema. Los pasajeros no tardan ni un segundo en recuperar la calma, se ríen, sintiéndose estúpidos de haberse asustado tanto. En unos minutos, el incidente está olvidado. El personal de cabina ofrece vino: -No quiero nada, gracias- dice una joven, -ya no bebo -. En la cabina de pilotaje, el piloto toca el panel de instrumentos, encuentra el botón del piloto automático y lo pone en funcionamiento. Entonces dice al copiloto: Algún día, estos hijos de p. van a gritar demasiado tarde y nos vamos a matar todos....

Futhark 7 (2012)

Castro, Secuencia y delimitación, 85-114

ISSN $1886-9300$ 
- Así la negación en alemán de un determinado nombre implica que en castellano se niegue el verbo: nada quiero $=$ no quiero nada = "Ich will nichts" o "Ich möchte nichts".

- Las construcciones en las cuales la negación cuantitativa se refuerza mediante "ni" o el "no", p. ej. "no tardan ni un segundo en recuperar la calma" = "es dauert keine Sekunde". No obstante, la lengua alemana ofrece una alternativa a la secuencia, la cual consistiría en el empleo de la paráfrasis verbal acompañada de adverbio: "Die Passagiere beruhigen sich sogleich".

- Ciertas expresiones neutras con negación implican anterioridad: "Ya no bebo vino" expresa la idea "antes bebía" = "früher habe ich getrunken" $\neq$ "heute habe ich schon genug getrunken", idea contraria a su aspecto actual a "por hoy ya he bebido bastante" = "heute habe ich schon genug getrunken". Podríamos considerar, por tanto, que el metateorema de las dos frases $X \leftrightarrow Y$ y $(X \rightarrow Y) \&(Y \rightarrow X)$ no es bicondicional.

En la psicología cognitiva es un tópico la pregunta acerca de cómo producimos y comprendemos textos o discursos. Y parece evidente que para ambos procesos construimos en forma activa distinto tipo de representaciones cognitivas (esto es, rasgos, significados, conjuntos estructurados de elementos). El objetivo final de la cognición es construir teorías inherentes que puedan explicar cómo las personas construyen y emplean las representaciones cognitivas. Así pues, las primeras teorías cognitivas de la comprensión y la producción del discurso deben enmarcarse en teorías generales de la cognición. De ello se deriva que la lingüística del texto se vea inspirada por teorías del discurso pertenecientes a otras disciplinas, como la pragmática de Van Dijk (2008), Grice (en Devitt, 2004) o Halliday y Hasan (2001), sin olvidar la inteligencia artificial, desarrollada por Schank y Abelson en el año 1977 (cf. Virtanen, 2004). Lo que parece evidente es que este hecho implica que esta teoría sea necesariamente compleja debido a que los textos y los discursos involucren varios niveles y componentes. En este sentido, bastaría comprobar cómo el relato del texto 1-"El viaje" contrasta con el texto 2 -"Hymnen an die Nacht" un fragmento escrito por Novalis para la revista "Athenäum" (en 1779): el lenguaje poético Futhark 7 (2012)

Castro, Secuencia y delimitación, 85-114

ISSN $1886-9300$ 
se engrandece con la mirada contemplativa a la naturaleza y las metáforas empleadas, las cuales aumentan el grado de dificultad en la comprensión del texto:

[Texto 2]

Texto en lengua original o L1 - "Hymnen an die Nacht"

Welcher Lebendige, Sinnbegabte, liebt nicht vor allen Wundererscheinungen des verbreiteten Raums um inn, das allerfreuliche Licht -mit seinen Farben, seinen Stralen und Wogen; seiner milden Allgegenwart, als weckender Tag. Wie des Lebens innerste Seele athmet es der rastlosen Gestirne Riesenwelt, und schwimmt tanzend in seiner blauen Flut athmet es der funkelnde, ewigruhende Stein, die sinnige, saugende Pflanze, und das wilde, brennende, vielgestaltete Thier -vor allen aber der herrliche Fremdling mit sinnvollen Augen, dem schwebenden Gange, und den zartgeschlossenen, tonreichen Lippen. Wie ein König der irdischen Natur ruft es jede Kraft zu zahllosen Verwandlungen, knüpft und löst unendliche Bündnisse, hängt sein himmlisches Bild jedem irdischen Wesen um. -Seine Gegenwart allein offenbart die Wunderherrlichkeit der Reiche der Welt ${ }^{9}$.

Otro ejemplo aplicado a los componentes nos lo ofrece este mismo autor, quien nos propone la obra versionada del

\footnotetext{
${ }^{8}$ El fragmento, perteneciente en concreto, a la primera parte de su obra "Die Erwartung" / "La esperanza", ofrece una visión metafórico-alegórica de reflexión ante la vida y la muerte, que el joven autor representa mediante el día y la noche. El día simboliza la luz, la alegría, representa el color, la luminosidad. El autor refleja en el día lo positivo, la belleza de la vida en una búsqueda o visión de la amada perdida, Sophie von Kühn, que muere tempranamente.

9 Novalis emplea verbos antitéticos. Por ejemplo "knüpft und löst", verbos que representan oposición entre sí. Por una parte "knüpfen" en el sentido de "unir". Por otra parte "lösen", que significa "desunión". Texto en lengua de destino o L2 - "Himnos a la noche": ¡Qué ser vivo, dotado de talento y sentido común, no ama ante todas las apariciones maravillosas del espacio que le rodea, la alegre luz -con sus colores, sus destellos y curvaturas; suave omnipresencia, cual despuntar del día. Como el alma más profunda de la vida respira el inmenso mundo de infatigables constelaciones y bailando nada en su azul marea, -respira como la planta sensual y absorbente de la piedra brillante, eterna, y el animal salvaje, ardiente, multiforme -pero ante todo ello la atenta mirada del hombre, su majestuosa forma de andar y su dulce tono de voz!.

Futhark 7 (2012)

Castro, Secuencia y delimitación, 85-114
}

ISSN $1886-9300$ 
"Rolandslied", escrita sobre el año 1170 por el clérigo Konrad. ${ }^{10}$ En estas ocasiones, nos transmite Ibáñez (2007: 81-100), nuestro nivel de comprensión y análisis es mayor si hay conocimiento de la obra originaria. ${ }^{11}$ Así, por ejemplo, el lector de la primera canción heróica de alabanza reparará en una concepción más objetiva de los acontecimientos, en una representación más sobria de Carlomagno, etc... ${ }^{12}$

Sin ir más lejos, los mismos autores literarios nos transmiten con frecuencia obras ya versionadas. Así la del "Romance del Conde Alarcos", anónima y del siglo de oro, en la que prácticamente se siguen los mismos patrones de quien fuera el creador del romance, pues el hilo argumentativo no experimenta grandes cambios: Dios castiga en ambas obras al rey conspirador y a la infanta.

\section{La plausibidad de cláusulas proposicionales}

Un enfoque para la representación semántica del texto que ha sido el fundamento de distintas teorías cognitivas, entre ellas las de Clark y Kintsch sobre la producción y la comprensión es el proposicional (en Feldman y Ballard, 2002): una proposición es una unidad teórica que contiene un predicado. Y toman como ejemplo un verbo o un adjetivo y un conjunto de argumentos asociados, tomándo como ejemplo sustantivos, cláusulas; estos argumentos desempeñan además determinados papeles semánticos de agente, paciente, locación, etc.

\footnotetext{
${ }^{10}$ En su queja a la noche el escritor muestra su disconformidad mediante reiterantes preguntas retóricas: Was quillt auf einmal so ahndungsvoll unterm Herzen, und verschluckt der Wehmut weiche Luft? Hast auch due in Gefallen an uns, dunkle Nacht? Was hälst du unter deinem Mantel, das mir unsichtbar kräftig an die Seele geht? Köstlicher Balsam träuft aus deiner Hand, aus dem Bündel Mohn. Die schweren Flügel des Gemühts hebst du empor.

${ }^{11}$ Precursora esta obra de la siguiente, y última de este autor, en la que se encarna la figura del cantautor-poeta que vivió durante el siglo XIII, Heinrich von Ofterdingen.

${ }^{2}$ El planteamiento de la obra gira en torno a la expulsión de los moriscos de España. En ella el emperador es descrito como -"der grimmige Karl" / "el furioso Carlomagno". Originariamente la canción era una alabanza al Emperador, como representante de Dios en la tierra, cuyos valores tenían su origen en el cristianismo y en el reflejo de la religión del Islam como equívoca.
}

Futhark 7 (2012)

Castro, Secuencia y delimitación, 85-114

ISSN $1886-9300$ 
Según este enfoque, los textos pueden segmentarse en proposiciones, en tanto son consideradas unidades funcionales principales. Al poner a prueba con el alumnado en la Asignatura de Gramática Contrastiva la plausibilidad psicológica de las representaciones proposicionales, confirmamos el papel relevante de las mismas en la memoria, la dificultad del procesamiento dificultad de comprensión, el tiempo de lectura medido), etc ${ }^{13}$.

Conforme a este mismo enfoque, la comprensión de textos puede concebirse como una secuencia de fases de procesamiento específicas cuyo resultado final es una representación mental semántica completa. En este sentido, algunas de las teorías aportadas por Minsky Rumelhart y Ortony son hoy en día muy aceptadas (cf. Peronard y Gómez Macker 2005: 19-32). Desde un punto de vista psicolingüístico estos campos se caracterizan porque las estructuras de conocimiento y los procesos de inferencia asociados "son empleados en representación de un significado de conceptos en el marco de un dominio particular", es decir, a objeto de precisar qué progreso experimentan las personas a la hora de procesar y comprender determinados contextos y / o discursos (cit. en Peronard y Gómez Macker 2005: 19-32) ${ }^{14}$. Este hecho enlaza directamente con la representacion conexionista de procesamiento del discurso, punto de vista desde el cual las proposiciones son unidades de representación de significado cuyos contenidos son

\footnotetext{
${ }^{13}$ Asignatura perteneciente al Plan de estudios de 18 de Abril de 1997.

${ }^{14}$ Podríamos decir que el conexionismo abarca dos posturas: - la primera de ellas se basa en la Inteligencia Artificial, entendida ésta como las redes que persiguen reproducir el funcionamiento real del cerebro e intentan, sencillamente, imitar competencias típicas del ser humano. La tecnología nos ofrece la posibilidad de construir arquitecturas que se pueden implantar en los denominados sistemas físicos no biológicos, entendidos como tales los ordenadores y los robots, facultados para reconocer textos, el habla o tipos de habla en entornos amigables, de discordia, etc...). - La segunda de ellas de basa en la biología: se persigue con ello construir redes que reflejen el mecanismo y la estructura real de nuestro sistema nervioso, esto es, la teoría conexionista apela al cerebro para delimitar o explicar competencias adjudicadas en un primer momento a la mente, como son el lenguaje, la percepción, y la memoria, rozando aquí el terreno de la psiconeurología y, derivando sus hallazgos a la Inteligencia Artificial. De ello se deduce que estas teorías conexionistas dependan ineludiblemente de esta disciplina, especialmente en las cuestiones relativas al sistema nervioso). Línea de investigación en la que incluimos a dos de los más relevantes exponentes en el campo de la reflexión teórica sobre el sentido, valor y límites del conexionismo, los Churchland.
}

Futhark 7 (2012)

Castro, Secuencia y delimitación, 85-114

ISSN $1886-9300$ 
conceptos lexicales completos, y no "primitivos semánticos" (cf. Rumelhart, 1996: 194-281). Estos autores clasificaban dichos conceptos proposicionales en argumentos -entidades sobre las que versa el texto- o predicados -conceptos con carácter relacional y de modificadores-. Estos conceptos interconectados ya habían sido objeto de estudio en 1971 por la gramática de caso de Fillmore.

Tomando como referencia el texto 3 -Ein Deutsches Réquiem- de J. Brahms, observamos que la aplicación de los niveles de coherencia en traducción pueden resumirse en la sustitución de oraciones que expresan temporalidad por oraciones compuestas por coordinación explicativa, lo que en la mayoría casos favorece la fluidez del texto en castellano:

[Texto 3]

Texto en lengua original o L1

Ein deutsches Requiem

I.

Selig sind, die da Leid tragen, denn sie sollen getröstet werden. (Matthäus 5.4)

Die mit Tränen säen, werden mit Freuden ernten. Sie gehen hin und weinen und tragen edlen Samen und kommen mit Freuden und bringen ihre Garben.

(Psalm 126, 5 und 6)

\section{Texto en lengua de destino o L2}

II.

Denn alles Fleisch, es ist wie Gras und alle Herrlichkeit des Menschen wie des Grases Blumen.

Das Gras ist verdorret und die Blume abgefallen.

$$
\text { (1. Petrus 1, 24) }
$$

So seid nun geduldig, liebe Brüder, bis auf die Zukunft des Herrn.

Siehe, ein Ackermann wartet auf die köstliche Frucht der Erde und ist geduldig darüber, bis er empfange den Morgenregen und Abendregen.

So seid geduldig.

(Jakobus 5, 7) 


\author{
Un Réquiem Alemán \\ I. \\ Bienaventurados los que padecen, \\ pues ellos serán consolados. \\ (Mateo 5.4) \\ Los que siembran con lágrimas, \\ recogerán con alegría. \\ Se han ido y lloran, \\ y portan la noble simiente, \\ y retornan con júbilo, \\ $y$ traen sus gavillas. \\ (Salmo 125, 5 y 6)
}

\section{II.}

Entonces toda la carne, es como la hierba y todo el esplendor del hombre es como la flor de los prados.

La hierba está seca

y la flor está marchita.

(Primera epístola de S. Pedro 1, 24)

Así, amados hermanos,

sed pacientes y esperad

la venida del Señor.

Mirad al campesino que

aguarda el fruto precioso de la

tierra y espera paciente la llegada

de la lluvia del otoño

y la primavera.

Así, sed pacientes.

(Epístola de Santiago, 5, 7)

En el caso de secuencias pronominales del alemán "und ist geduldig darüber" / "y espera paciente", se ha omitido intencionadamente en castellano la traducción de "darüber", con vistas a la consecución de un mayor grado de estilismo. En los contrastes "ist wie..." / "wie" no observamos divergencias de importancia, sí mencionables como parte funcional del análisis. En este sentido son también dignas de mención las oraciones relativas que encabezan el texto y el segundo párrafo del mismo, respectivamente, y que se manifiestan en cuanto a su estructura a modo de substantivación: "Selig sind, die da Leid tragen", "Die mit Tränen säen". Además se observa que el discurso se agiliza en lengua alemana en los casos en que emplean oraciones pasivas con verbos modales, en este caso "getröstet werden" acompañado de "sollen", y se contribuye así a la objetividad, entendida desde el 
punto de vista del autor como emisor que goza de la perspectiva de explicitar un mensaje. Así lo expresa también E. Werlich:

\begin{abstract}
"Das Passiv wird darum vor allem dann in Textformen gewählt, wenn der Sprecher den Handlungsträger nicht nennen will oder nicht benennen kann. Das ist der Grund, warum die Sprecherperspektive des Passivs vor allem in jeden Textformen verbreitet ist, die durch objektive Präsentation gekennzeichnet sind."
\end{abstract}

\title{
(cit. en Pilar Elena 1994)
}

En líneas generales, en el texto destacan los denominados "conectores del discurso", que pertenecen a las formas de secuencia de textos expositivos, tales como "denn", "und" o wie. De entre los elementos destacables que intervienen en este texto destacamos tres descriptivos fundamentales:

a. El observador: la posición del observador en el texto se sitúa fuera de la escena o cuadro o fuera de él · En tercera persona en este caso, en otras ocasiones en primera persona. Fija en nuestro texto y / o en movimiento en los demás casos.

b. El mundo real o imaginario: los objetos o elementos a describir son paisajes, ambientes interiores o exteriores, personas, objetos, animales. Los elementos pueden estar quietos, en movimiento o ambas cosas a la vez: "Sie gehen hin und weinen und tragen edlen Samen und kommen mit Freuden" / "Se han ido y lloran, y portan la noble simiente, y retornan con júbilo, y traen sus gavillas". Asimismo pueden verse en forma parcial y restrictiva ("Selig sind, die da Leid tragen" $\rightarrow$ "Bienaventurados los que padecen"; "Die mit Tränen säen" $\rightarrow$ "Los que siembran con lágrimas"), en su totalidad ("alles Fleisch" / "toda la carne"; "alle Herrlichkeit des Menschen" / "todo el esplendor del hombre") o en detalle ("Das Gras ist verdorret und die Blume abgefallen" $\rightarrow$ "La hierba está seca y la flor está marchita").

c. Recursos: se emplean gran cantidad de recursos, de los cuales algunos son propios o característicos de esta tipología textual. El manejo del lenguaje es muy detallado. En textos de tipo descriptivo-literario, se persigue un fin estético, por lo que el despliegue de recursos es aún mayor. Enumeramos a continuación dichos recursos:

Futhark 7 (2012)

Castro, Secuencia y delimitación, 85-114

ISSN $1886-9300$ 
- Profusa adjetivación: P. ej. "con alegría" > "mit Freude"; "la noble simiente" < “...und tragen edlen Samen"; "la hierba está seca" > "das Gras ist verdorret"; "die Blume ist abgefallen" < "la flor está marchita", en forma de oraciones pasivas de estado o Zustandspassivsätze. Además de "geduldig" > "pacientes", "die köstliche Frucht" > "el fruto precioso".

Reproducción de imágenes visuales. Un ejemplo lo constituye [...] "Die mit Tränen säen" hasta [...] "und bringen ihre Garben".

- Reproducción de otras imágenes auditivas, táctiles, gustativas, olfativas.

- Comparaciones: P. ej.: "es ist wie Gras"; "wie des Grases Blumen“.

- Metáforas: P. ej. "mit Tränen säen werden mit Freuden ernten"; "tragen edlen Samen und bringen ihre Garben".

Al modelar los procesos de comprensión de discurso, los investigadores comparten como un supuesto básico que algunas piezas del texto pueden ser entendidas en la medida en que pueden ser relacionadas con un estereotipo situacional -frame o script(Smolenski, 2008: 1-43). De ese modo distinguen entre scripts, p. ej. "un restaurante", pistas "café" o "fast food", y roles, que estarían conformados por palabras como "hamburguesa" o "pescado". En nuestro texto, el script lo conformaría el "paisaje", pistas podrían ser "el Señor", "el esplendor del hombre" y roles serían "los hombres que padecen". Los denominados "esquemas" son representaciones complejas, o de "nivel más alto", que refieren a conceptos genéricos de todo tipo: objetos, situaciones y secuencias de eventos y acciones. Esta visión parece un enfoque fiable para analizar la comprensión textual.

\subsection{Ontogénesis del género discursivo}

De todo ello se deriva que escritor/lector ha de construir cognitivamente a través de complejos procesos ontogenéticos en su interacción con el medio físico, social y cultural. Esto quiere decir que el sujeto debe elaborar representaciones cognitivas de objetos

Futhark 7 (2012)

Castro, Secuencia y delimitación, 85-114

ISSN 1886-9300 
variados, procesos y mecanismos de diversa índole, almacenándolos en sus diversos sistemas mnemónicos.

Mediante los denominados "procesos de experiencias de interacción informal y otros de educación formal y sistemática", parte de estos objetos externos son conocidos y pasan a ser representados en la memoria de un individuo, manipulados o no. Ibánez califica estos constructos como altamente dinámicos, evolutivos y variables a lo largo del tiempo y de las posteriores interacciones del sujeto con entornos y experiencias variadas. Muchos de estos constructos son los que dan forma y constituyen lo que denomino "géneros discursivos de producción". Enmarcado en este contexto que se describe, el texto específico presenta una determinada intencionalidad y ésta es recibida por el lector de las maneras más diversas. A este fin emplea diversos constructos sintácticos. Pongamos como ejemplo la moralidad tragi-cómica del "Elckerlijc" neerlandés (1510), atribuida a Peter Van Diest. La representación se abre con un monólogo de Dios, quien expresa sus quejas por la avaricia del hombre. "Elckerlijc" / "Todohombre" representa la figura de cada uno de nosotros. El texto de tono moralizante-didáctico, nos presenta un determinado modelo de conducta o modo de vida, el cual debemos seguir si queremos obtener la salvación ${ }^{15}$. El texto original de la obra y la traducción del "Elckerlljc" / "Todohombre" se caracteriza, principalmente, por sus intencionales giros sintácticos, p. ej.: "Al ghevet u vreemmt, het moet gescieden" / "Un viaje has de emprender no menos presto" /, "debes cuentas rendir", etc.

Desde esta propuesta, podemos deducir que el mundo externo se constituye en un punto de partida para la construcción relativa e intersubjetiva de los conocimientos individuales. Esta afirmación reafirmaría que la vinculación de la producción y comprensión discursiva-textual queda sujeta al proceso de ontogénesis y que incide muy directamente en la labor traductológica. De modo más específico, en lo que atañe al género del discurso, los participantes y sus roles sociales, queda claro que

\footnotetext{
${ }^{15}$ El tono didáctico de "Elckerlijc" nos recuerda a la temática presente en la obra de don Juan Manuel, en la que se reflejan todos los estados y estratos sociales - ricos y pobres, nobles y plebeyos, mercaderes, frailes, burgueses y prelados.
}

Futhark 7 (2012)

Castro, Secuencia y delimitación, 85-114

ISSN 1886-9300 
ellos existen "allí afuera" (cf. Fodor y LePore 2001: 328-343). No obstante, dado que el género es una herramienta discursiva de interacción social, debe necesariamente existir una representación cognitiva de esos participantes y de sus potenciales roles para que el escritor pueda transmitir las funciones comunicativas que se ponen en juego a través del lenguaje y el hablante pueda comprender el significado del texto (cf. Sopena 2008: 20-30). Véase:

\section{[Texto 4]}

\section{Elckerlijc (s. XV)}

\section{Die Doot}

Jae ikc, certayn!

Al hebdi Sijns vergheten, alst blijct,

Hi peynst wel om u in Sijn rijck,

Alsoe ick u sal voer oghen legghen.

\section{Elckerlijc}

Wat begheert God van my?

\section{Die Doot}

Dal sal ic di segghen:

Reckeninghe wilt $\mathrm{Hi}$ van u ontfaen

Sonder eenich verdrach.

\section{Elckerlijc}

Hoe sal ic dat verstaen?

Reckeninghe? Wat sal bedieden?

\section{Die Doot}

Al ghevet $\mathrm{u}$ vreemmt, het moet gescieden.

Oec moetti aen nemen sonder verdrach

Een pelgrimagie, die niemant en mach

Weder keeren in gheender manieren.

Brengt u gescriften ende u pampieren

Met $u$, ende oversietse bedachtich,

Want ghi moet voer God almachtich

Rekenninghe doen, des seker sijt,

Ende hoe ghi bestaet hebt uben tijt,

Wan uwen wercken, goet ende quaet.

ISSN $1886-9300$ 
En obras más actuales es ciertamente el contexto el que eventualmente puede activar estos conocimientos, pero si no existe una construcción previa de ellos almacenada en la memoria del sujeto, la interacción social fracasará en su objetivo comunicativo (cf. Charaudeau, 2004: 23-39) ${ }^{16}$.

\subsection{Micro y macroestructura}

Para responder a la pregunta acerca de cómo se interrelacionan las proposiciones y las correspondientes representaciones proposicionales de manera coherente, Kintsch y Van Dijk habían desarrollado anteriormente sucesivos modelos que identificaban los tipos de coherencia y que especifican cómo se construyen estructuras textuales coherentes en una memoria de trabajo limitada. Kintsch y Van Dijk distinguieron los dos niveles de coherencia más conocidos por la lingüística textual (cit. en Mannes y Doane 2007: 61-87):

1. La microestructura textual, que se define como el nivel que conecta proposiciones textuales explícitas mediante

\footnotetext{
${ }^{16}$ Ejemplos son "De zeven laatste zinnen" / "Las últimas siete palabras" (2010), "Hoe ik nimmer de Ronde van Frankrijk loor min-twaalfjarigen won (en dat het me spijt) / "Nunca gané el Tour de Francia para menores (y cuánto lo lamento)" de Ivo Victoria (2009), y Dorsvloer vol confetti "Tierra árida cubierta de confeti" (2009), Vaslav (2010) se presentan tres temáticas diferentes: - en la primera, se hace "un recorrido por la insignificante vida" de un padre alcohólico "una miga en el cosmos" en el no entenderíamos la reflexión que hace Dimitri Verhulst sin conocer la historia de la humanidad conforme a la tradición cristiana; - en la segunda, la infancia y adolescencia del protagonista, nos conducen a una gran mentira.explicativa; la tercera nos adentra en la holanda de los años 80 a través de una serie de escenas, en el sentido más cinematográfico de la palabra, mediante las cuales la autora, Franca Treur, se sirve para adentrarnos en la sociedad del momento, en la era de la cuota lechera y de las tasas suplementarias, en los círculos calvinistas y en una especie de autoridad catártica de la congregación eclesiástica; - en la cuarta de ellas, Vaslav, de Arthur Japin, nos plantea como temática "la importancia de saber caer", reflejándonos los momentos de gloria del bailarín Vaslav Nijinsky, sumergido en las profundidades de su locura, alrededor de las tres personas más importantes dentro de su vida: Sergéi Diágilev, su mujer, Romota de Pulsky y Meter, un sirviente que poco a poco asume las funciones de cuidador personal y puede que de amante. Sin entender las complicadas relaciones que este triángulo desencadenan el escándalo de "Le Sacre du Printemps" en 1913, tampoco comprenderíamos el famoso salto que Nijinsky realiza en una única ocasión al aire libre, en el campo, en una función privada para su hija y su ayudante, Peter. Más adelante, es el propio Nijinsky quien resume su drama personal en una sola frase: «Lo difícil es volver a bajar».
}

Futhark 7 (2012)

Castro, Secuencia y delimitación, 85-114

ISSN $1886-9300$ 
relaciones entre argumentos o conexiones conceptuales como temporalidad o causalidad. Tales conexiones locales se establecen, además, mediante distinto tipo de relaciones funcionales. En este breve texto paródico, en el que prácticamente la continuación del ejemplo citado entre paréntesis es la referencia a lo anteriormente explicitado, las relaciones funcionales se expresan mediante el contraste, la comparación, la ejemplificación, la explicación, etc... Aquí las proposiciones constituyen la microestructura del textual pendiente de interpretación (cf. Dolan y Dyer):

\section{[Texto 5]}

Doofe Leute sollten Schilder tragen müssen, auf denen steht: "Ich bin doof". [Dann würde man sich nicht auf sie verlassen, oder? Du würdest sie nichts fragen. Es wäre wie " tschuldigung, ich... äh, vergiss es. Hab das Schild nicht gesehen." Man wüsste zumindest, was auf einen zukommt. So wie letztes Jahr, als ich mit meiner Familie mitten im Umzug stand. Die ganze Wohnung voll mit Umzugskartons, der Lkw vor der Tür. Mein Nachbar kommt rüber und fragt: "Hey, du ziehst um?" "Nö. Wir packen nur ein- bis zweimal die Woche unsere Klamotten ein, um zu sehen, wie viel Kartons wir dafür brauchen. Hier ist dein Schild!"].

2. McKoon y Ratcliff definen la macroestructura textual como el nivel que vincula segmentos más amplios del texto en virtud del conocimiento de mundo y de los esquemas genéricos, como los "guiones" -antes denominados scripts-, el conocimiento mismo del género, la distribución informativa y los esquemas de textualización narrativo, expositivo y argumentativo (cf. Heinemann 2006: 2-29).

Rumelhart aduce que, por un lado, la comprensión de texto consiste en buscar la configuración de esquemas que den cuenta adecuadamente del significado de tal texto (Rumelhart, 1980). Por otro lado, los esquemas activos durante la comprensión permiten rellenar los denominados "valores ausentes" (cfr. Smolensky, McClelland y Hinton, 1986).

Un importante déficit de la teoría conexionista consiste en cómo pueden mostrarse los esquemas con información ausente y que la información fuera interpretada correctamente. Los Chunks de

Futhark 7 (2012)

Castro, Secuencia y delimitación, 85-114

ISSN $1886-9300$ 
conocimiento conexionista han intentado explicar esquemas que solucionen problemas de Inteligencia Artificial, sin dejar de ser vagas especificaciones de elementos co-ocurrentes de un modo esperado o de un objeto del esquema.

\section{Inferencia de modelos mixtos: input /output}

Durante la década de los 80 los modelos cognitivos pusieron mucha atención en las propiedades del texto explícito, pero, a raíz de la crítica a esta postura, las investigaciones fueron paulatinamente dando mayor importancia al lector -a fin de cuentas intérprete del texto- y a las restricciones de la cognición en general. La comprensión se concibió como un proceso de construcción activo, flexible y estratégico (cfr. Van Dijk y Kintsch, 1983; Graesser, 1981) y comenzaron a crearse modelos computacionales que simulaban los mecanismos cognitivos. Surgieron modelos matemáticos, los cuales cuantificaban patrones precisos de los tiempos de procesamiento, estableciendo límites de memoria ${ }^{17}$.

Según la mayoría de los autores, el significado matemático conseguiría distribuirse a través de toda la red (neuronal):

"La representación de significado consiste en la activación de los valores correspondientes a las unidades que captan el contexto y la entrada -que podemos denominar input-; esta idea contrasta nítidamente con la posición simbólica, según la cual el significado se localiza en una expresión simbólica o en un grupo de expresiones simbólicas".

(Graesser, cit. en A. Devitt 2004)

En la actualidad los modelos construcción-integración, $\mathrm{Cl}$, de Just y Carpenter (cit. en Devitt 2004) y Kintsch (2006: 163-182) son

\footnotetext{
${ }^{17}$ Algunos de los modelos de red no aportaban soluciones sobre el modo en que las personas recuperan información relevante desde la memoria. Este hecho implicaba que las propuestas anteriores tuvieran su fundamento en la "pura asociación". Con el fin de poner término a esta problemática, algunos enfoques han propuesto redes complejas de conocimiento específico de dominio. Las estructuras altamente organizadas más actuales, tales como los "frames" de Minsky o los esquemas de Rumel- asumen que la información que reside en la memoria está dotada de estructura interna.
}

Futhark 7 (2012)

Castro, Secuencia y delimitación, 85-114

ISSN $1886-9300$ 
los más destacados dentro del ámbito textual. Ambos modelos se basan en un sistema de producción colaborativo basado en la activación y ambos son describibles como híbridos de teorías simbólicas y conexionistas ${ }^{18}$.

Estos interesantes modelos $\mathrm{Cl}$ establecen en realidad tres niveles divergentes de representación:

- Forma de superficie, constituida por las palabras exactas y sintaxis de las oraciones.

- La base textual proposicional, esto es, conexiones microestructurales entre proposiciones.

- El modelo situacional-referencial, el cual integra la información del texto con el conocimiento de mundo del lector y así hace referencia al mundo único del texto particular.

Obviamente se deduce que el modelo $\mathrm{Cl}$ es híbrido, pues combina expresiones simbólicas (entre las que se cuentan palabras con contenido -las más significativas entre ellas, verbos, sustantivos, adjetivos-, proposiciones textuales explícitas y conocimiento implícito de mundo relevante al texto) y vínculos conexionistas, que se especifican de acuerdo con las restricciones de la forma de superficie, la base del texto y el conocimiento explícito de mundo.

\footnotetext{
${ }^{18}$ El hilo conductor del conexionismo tiene por objeto principal analizar los niveles denominados subsimbólicos, tales como esquemas, sistemas de producción, etc., y que son utilizados por el paradigma cognitivo. Los conexionistas mantienen que la representación mental no puede ser explicada simple y llanamente apelando a la analogía con las características computacionales de una máquina. A diferencia de los enfoques proposicionales simbólicos más clásicos, los cuales manifiestan que el conocimiento es localizable y se encuentra almacenado, aquí sería el resultado de la activación de una red de conexiones entre unidades muy simples (similares, pero no identificables, a las neuronas). En las redes conexionistas, la representación de conocimiento tiene lugar a través de redes formadas por unidades y conexiones.
}

Futhark 7 (2012)

Castro, Secuencia y delimitación, 85-114

ISSN 1886-9300 
"El modelo de $\mathrm{Cl}$ simula la fluctuación dinámica de los valores de activación de las unidades en la red: los valores cambian a medida que avanza la comprensión, palabra por palabra, proposición por proposición y oración por oración”.

(cit. en K. Hylan 2008: 41-62)

Para delimitar estos modelos aplicados a la lengua alemana y castellana vamos a partir del siguiente texto:

[Texto 6]

Texto en lengua original o L1 - "Aber Frau Feldbauer..."

"Gude Morsche," begrüsste Emma Feldbauer die Bäckersfrau and auch die Frau Direktor Wunderlich die gerade eine wunderschöne Schokoladentorte in Empfang nahm. "Ei was is des awer en scheene Kuche Fra Diregdor," staunte Emma, "für wen hawe Se dann den bagge losse?" "Für meinen Mann natürlich. Der hat nämlich heute Geburtstag." "Ei do werd der sich awer freue. Moin Mann, de Fritz, hat jo aach bald Geburdsdaach. Dem kennt ich jo aach emol su ebbes bestelle." "So? Wie alt wird er denn?" "Ei, der werd schun drei un dreissisch." "Das kann aber nicht ganz stimmen," meinte die Frau Direktor. "Ihr Mann muss doch älter sein. Ihre Liesel ging doch in die gleiche Schulklasse wie unsere Gisela. Und die ist schon fast zwanzig." "Ach ja, des schdimmd jo. Also glaawe Se, des hatt isch schun ganz vegesse. Do muss moin Fritz schun drei un ferzisch wern. Jesses, wie doch die Zeitvegieht, gell?" [...].

Texto en lengua de destino o L2 - "Pero Sra. Feldbauer..."

"Buenos días," Emma Feldbauer saludó a la panadera y a la Sra. directora Wunderlich, que en ese preciso momento recogía una estupenda choco-tarta. "Qué pastel tan estupendo Sra. Directora!", prosigió Emma sorprendida, "¿Para quién ha encargado el pastel?" "Es para mi esposo, que precisamente hoy cumple años". "Qué contento se pondrá". Mi esposo, Fritz, también cumplirá pronto. Le podría encargar también una parecida". "¿Ah sí?, ¿Y qué edad tiene?" "Ah, pues cumple ya treinta y

Futhark 7 (2012)

Castro, Secuencia y delimitación, 85-114

ISSN 1886-9300 


\begin{abstract}
tres ". "¡Cómo va a ser!", opinó la Sra. directora. "Vamos, su esposo debe ser bastante mayor". Nuestra hija, Liesel, iba a la misma clase que la nuestra, Gisela. Y ella ya casi tiene veinte años...". "Ah, pues es verdad. Se me había olvidado por completo. Entonces Fritz debe de estar a punto de cumplir los cuarenta y tres". ¿Jesús, cómo pasa el tiempo!, ¿eh?! [...].
\end{abstract}

En el ciclo computacional de comprensión del texto que describimos las palabras activan algunas unidades y esa activación se difunde a través de la red hasta que eventualmente se estabiliza; éste lo identifica Pylyshyn (1968: 3-61) como un proceso recurrente. En este caso, el valor de activación de cada unidad puede delimitarse como una función de la secuencia de ciclos de comprensión. En traducción mecánica estos ciclos, se acentúan, sobre todo, en el caso de palabras que puedan resultar llamativas, como por ejemplo "choco-tarta", en lugar de "tarta de chocolate" y se constata en con los giros y expresiones dialectales "was is des awer en scheene Kuche", "für wen hawe Se dann den bagge losse?" (cf. Castro Moreno 2007).

En general, podemos afirmar que las unidades que tienen vínculos de conexión positivos se asientan en valores altos de activación y, por el contrario, aquellas unidades que estén separadas de las otras causarían un efecto de activación bajo. En este sentido podemos incluir palabras con connotaciones positivas, divertidas o que acerquen a los interlocutores: ¡Jesús, cómo pasa el tiempo!, ¿eh? < Jesses, wie doch die Zeit vegieht, gell?".

\begin{abstract}
Así el modelo $\mathrm{Cl}$ es capaz de predecir patrones de datos con experimentos psicológicos: por ejemplo, cuando se les pide a los lectores que recuerden un texto con posterioridad a su lectura, es claro que la posibilidad de recuerdo de las distintas proposiciones es disímil. Las posibilidades de recuerdo están correlacionadas con valores de activación promedio en los ciclos de comprensión.
\end{abstract}

(cit. en I. Bruce 2008)

En cuanto al procesamiento del discurso, podemos añadir que la comprensión del lenguaje es un proceso donde un sujeto, al leer o al escuchar, descifra una oración en un conjunto de conceptos. Según J. Ezquerro, en la mayor parte de los casos,

Futhark 7 (2012)

Castro, Secuencia y delimitación, 85-114

ISSN $1886-9300$ 


\begin{abstract}
los procesos de comprensión consisten en el intento de hacer explícito lo que se encuentra implícito en una oración o situación.
\end{abstract}

(cit. en R. Ibáñez 2008:249-276)

Teniendo en consideración esta máxima, lo que se expresa en una oración es, en ocasiones, una pequeña parte de lo que el hablante o el escritor, en su caso, intenta transmitirnos. Lo normal consistiría en que al menos una parte considerable de la información conste implícitamente y que el trabajo de nuestra mente consista en suplir o completar lo que en ningún momento ha sido literalmente emitido. Para procesar los rasgos explícitos de un texto, el lector debe transmitir una cantidad considerable de conocimiento preexistente al proceso de comprensión de lectura (cf. Sharkey y Mitchell 2005: 253-270). La interacción entre procesos basados en texto y procesos basados en conocimiento, y entre niveles dentro de cada proceso es esencial para la comprensión de la lectura:

\begin{abstract}
La comprensión se ejecuta tanto de arriba a abajo como de abajo a arriba. La comprensión está dirigida tanto por conceptos preexistentes como por los datos producidos por el texto. Además, dado que el significado está determinado sólo parcialmente por el texto mismo, la lectura debe ser un proceso constructivo, inferencial, caracterizado por la formación y comprobación de hipótesis sobre lo que trata el texto.
\end{abstract}

(cit. en W. Kintsch 2008: 163-182)

Los autores Van Dijk, Kintsch y Johnson-Laird piensan que un enfoque combinado muestra que ambos tipos de representación contribuyen a la comprensión (cf. Bazerman 2008).

\title{
5. Conclusiones
}

Evidentemente, los procesos conexionistas, aplicados o no a la traducción, precisan de información adicional requerida, y ésta, según el modelo conexionista, proviene de la memoria y se incorpora de modo lógico durante el proceso de comprensión. De ello se deriva que aprendamos, siendo necesario, a realizar predicciones, hipótesis y conjeturas con respecto a lo que estamos diciendo. Este factor incide asimismo en la producción textual, Futhark 7 (2012) Castro, Secuencia y delimitación, 85-114

ISSN 1886-9300 
convirtiéndose así en recíproco. De algunos de estos datos de procesamiento nos hemos servido en las ejemplificaciones, concluidas:

(i) según el modelo neuronal que constituyen las teorías de conexión, a través de la cual el ser humano es capaz de procesar y almacenar información discursiva y textual a través de estados, definidos éstos como vínculos externos. A partir de las teorías simbólicas se ha definido una memoria de trabajo y un conjunto amplio de conceptos, proposiciones esquemas, a modo reglas de producción. Esto trae consigo que el lector, mediante la simple búsqueda léxica en un diccionario, considerando el contexto que subyace a una palabra y determina su significado, esté capacitado para identificar eventos derivados de la palabra en cuestión o acoplar conceptos en una descripción de evento o estado. En la traducción terminológica y textual estos parámetros tienen altos índices de interacción.

(ii) por procesos que guardan estrecha relación con el fenómeno de inferencia, mediante el cual tienen lugar a un mismo tiempo la identificación de eventos y la realización de conjeturas e hipótesis sobre lo que el escritor, lector, traductor o intérprete tiene en mente, pero que no expresa explícitamente. Este factor sería coincidente con las teorías conexionistas y simbólicas, pues somos capaces de procesar textos de forma activa, dinámica y ontogénica. El procesamiento, en nuestro modelo conexionista, abarca la producción y la comprensión textual. De la anterior teoría conexionista adoptamos que la información en la memoria de trabajo varía a lo largo del tiempo, es decir, varía, de ciclo de comprensión en ciclo de comprensión. De lo anterior deducimos que el proceso de aprender puede crear nuevos hechos y reglas de producción en la memoria a largo plazo, los cuáles podrían ser relevantes a la hora de su traducción. Además, añade Kintsch (2008) que este proceso se desdobla en otros dos subprocesos, igualmente relevantes: uno consistente en el relleno de espacios en blanco y en la identificación del objetivo, y otro consistente en la conexión de eventos. Este último proceso conecta el input actual con la información previa, por el cual los valores de activación de las unidades en la red computacional cambian a medida que avanza la comprensión, 
palabra por palabra, proposición por proposición y oración por oración, procedimiento similar al de nuestra mente.

Cabe pensar, por lo tanto, que en casos de desambigüación de sentidos de palabra en procedimientos textuales o discursivos, está altamente involucrado el contexto de conocimiento, mecanismos para hallar asociaciones entre palabras próximas, algunos mecanismos para manejar las claves de desambigüación sintáctica, mecanismos para manejar las negociaciones de selección -restricción-reconciliación- entre palabras ambigüas y, como último recurso, el ya mencionado recurso de inferencia. Por consiguiente, el texto o discurso debe ser considerado a varios niveles micro y macroestructurales, con unidades de análisis que van desde las letras hasta el texto como un todo, como un proceso interactivo multinivel.

A grandes rasgos, podemos afirmar tácitamente que el procesamiento textual es similar en muchos aspectos a la resolución de problemas. Los sujetos son constructos de la información contenida en el texto, es decir, son los que deben construir una representación de la información contenida. No obstante, es necesario señalar que la comprensión se dificulta en la medida en que emisor e interlocutor, ambos pilares imprescindibles en la mecánica productiva, no construyen la representación informativa adecuadamente a partir de elementos representacionales de situaciones reales descritas. En este caso, la comunicación se ve necesariamente interrumpida. Este hecho constata que inferencias y proposiciones son enmarcables dentro en un mismo parámetro textual, el microestructural.

\section{Referencias bibliográficas}

BAZERMAN, CH., Handbook of research on writing. History, society, school, individual text, Nueva York, Erlbaum, 2008.

BRUCE, I., Academic writing and genre. A systematic analysis, London, Continuum, 2008.

CASTRO MORENO, C., "La Hipotaxis como Absatzkomponent", en Estudios Filológicos Alemanes, 2006, Sevilla, Fénix Editora, 11, págs, 525540.

Futhark 7 (2012)

Castro, Secuencia y delimitación, 85-114

ISSN $1886-9300$ 
CRESPO, A., Cognición humana. Mente, ordenadores y neuronas, Madrid, Editorial Centro de Estudios Ramón Areces S.A., 2002.

CHARAUDEAU, P., "La problemática de los géneros. De la situación a la construcción textual", en Revista Signos, 2004, Universidad de Valparaíso, Ediciones Universitarias, 37(56), págs. 23-39.

DEVITT, A., Writing Genres, Carbondale, Southern Illinois University Press, 2004.

DOLAN, C. y DYER, M.G., "Symbolic schemata in connectionist memories: Role binding and the evolution of structure", en Technical Report, 2007, Los Ângeles, Universidad de California, Artificial Intelligence Laboratory, 87(11), págs. 303-340.

ELENA GARCíA, P., "Aspectos teóricos y prácticos de la traducción: Alemán-Español", Universidad de Salamanca, Ediciones de la Universidad de Salamanca, 1994.

FELDMAN, J.A. y BALLARD, D.H., "Connectionist models and their properties", en Cognitive Science, 2002, Universidad de Berkeley, Ediciones Universitarias, 6 (3), págs. 205-254.

FODOR, J.A. y LEPORE, E., "Why meaning (probably) isn't conceptual role", en Mind \& Language, 2001, Edinburgo, Journal of Evolutionary, 6, págs. 328-343.

$\neg$ y PYLYSHYN, Z.W., Connectionism and Cognitive architecture: A critical analysis, en Cognition, 1988, York, Ediciones Universitarias, 28, págs. 3-71.

HALLIDAY, M y HASAN, R., Language, Context and Text Aspects of Language in a Social - Semiotic Perspective, Oxford, University Press, 2001.

HEINEMANN, W., "Zur Diskussion um Basisklassen des Kommunizierens. Rückschau uns Ausblick", en Adamzik, K. (eds.) "Textsorten”, 2006, Tubinga, Stauffenburg, Brigitte Narr GmbH, 4, págs. 2-29.

HYLAND, K., "Academic clusters: text patterning in published and postgraduate writing", en International Journal of Applied Linguistics, 2008, Londres, Institute of Education, 18(1), págs. 4162.

IBÁÑEZ, R., "Cognición y comprensión: Perspectivas para un análisis crítico al trabajo investigativo de Rolf Zwaan", en Revista Signos, 2007, Valparaíso, Ediciones de la Universidad Pontificia, 40 (63), págs. 81-100.

Futhark 7 (2012)

Castro, Secuencia y delimitación, 85-114

ISSN $1886-9300$ 
$\neg$, "El Texto Disciplinar y el acceso al conocimiento desde el análisis del género: ¿Regulación del conocimiento o persuasión”, en G. PARODi (eds.), Géneros Académicos y Géneros Profesionales: Accesos discursivos para saber y hacer, 2008, Valparaíso, Ediciones de la Universidad Pontificia, 44(72), págs. 219-246.

ᄀ, PARODI, G. / VENEGAS, R. / GUTIÉRREZ, R.M., "Géneros del discurso en el Corpus PUCV-2006: Criterios, definiciones y ejemplos", en G. PARodi (eds.), Géneros Académicos y Géneros Profesionales: Accesos discursivos para saber y hacer, 2008, Valparaíso, Ediciones de la Universidad Pontificia, 44 (56), págs. 39-74.

$\mathrm{KINTSCH}$, W., "The role of knowledge in discourse comprehension construction-integration model", en Psychological Review, 2006, Colorado, American Psychological Association, 95, págs. 163-182.

$\neg$, Comprehension: A paradigm for cognition, Nueva York, Cambridge University Press, 2008.

LANGE, T. y DYER, M.G., "Frame selection in a connectionist model of highlevel inferencing", en Technical Report UCLA, 2009, Los Ángeles, Ediciones de la Universidad de California (UCLA), Computer Science Department, 15, págs. 303-318.

MANNES, J.M. y DOANE, S.M., "A hybrid model of script generation: or getting the best from both worlds", en Connection Science, 2007, Londres, Taylor \& Francis, 3, págs. 61-87.

PERONARD, M. y GÓMEZ-MACKER, L., "Reflexiones acerca de la comprensión lingüística: Hacia un modelo", en Revista de Lingüística Teórica y Aplicada, 2005, Concepción (Chile), Ediciones Universitarias, 23, págs. 19-32.

RUMMELHART, D. y MCCLELLAND, J., "Parallel distributed processing", en Studies in the microstructure of cognition, 1996, Cambridge, MIT Press, 1, págs. 194-281.

SMOLESNSKI, P., "On the proper treatment of connectionism", en Behavioral and Brain Sciences, 2008, Yale, Ediciones Universitarias, 11, págs. 1-43.

VAN DIJK, T., Discourse and context. A sociocognitive approach, Cambridge, University Press, 2008.

CHOMSKY, N., Una aproximación naturalista a la mente y al lenguaje, Madrid, Editorial Prensa Ibérica S.A., 1998.

ISSN 1886-9300 
VIRTANEN, T., Approaches to cognition through text and discourse, Berlin, Mouton de Gruyter, 2004.

SHARKEY, N.E. y MITCHELL, D.C., "Word recognition in a functional context: the use of scripts in reading", en Journal of Memory and Language, 2005, San Diego, Ediciones Universitarias, 24, págs. 253-270.

SHASTRI, L Y AJJANAGADE, V., "From simple associations to systematic reasoning: A connectionist representation of rules, variables, and dynamic bindings using temporal synchrony", en Behavioral and Brain Sciences, 2006, Yale, Ediciones Universitarias, 16, págs. 417494.

SOPENA, J.M., "Verbal description of visual blocks world using neural networks", en Technical Report UPB, 2008, Barcelona, Ediciones Universitarias, 50(10), págs. 20-30. 